\title{
Veränderungen der Beeinträchtigungsschwere und Zielerreichung bei analytischer Psychotherapie bei Kindern und Jugendlichen mit externalisierender Symptomatik
}

\author{
D. Fiedler · K. Weitkamp · G. Romer · S. Wiegand-Grefe
}

Eingegangen: 18. Januar 2016 / Angenommen: 21. Mai 2016 / Online publiziert: 21. Juni 2016

(c) Der/die Autor(en) 2016, korrigierte Publikation 09/2017. Dieser Artikel ist eine Open-Access-Publikation.

\section{Zusammenfassung}

Ziel Die vorliegende Arbeit untersucht Veränderungen der Beeinträchtigungsschwere sowie die Therapiezielerreichung durch analytische Psychotherapie bei Kindern und Jugendlichen mit externalisierenden Störungen.

Methodik Die Daten sind Teil einer kontrollierten Interventionsstudie mit prospektivem Studiendesign, wobei Beeinträchtigungsschwere und Therapiezielformulierung in dieser Untersuchung ausschließlich mittels eines Within-Designs analysiert wurden. Untersucht wurden 60 Kinder und Jugendliche zwischen vier und 17 Jahren $(M=11,25 ; S D=3,91 ; 22$ männlich) mit vorwiegend externalisierender Symptomatik, die in ambulanter analytischer Psychotherapie behandelt wurden. Erfasst wurde die Beeinträchtigungsschwere mit dem Beeinträchtigungsschwere-Score für Kinder und Jugendliche (BSS-K) sowie die Therapieziele und deren Erreichung im Therapeutenurteil.

Ergebnisse Am häufigsten benannten Therapeuten Interpersonale Ziele. Die Therapieziele wurden im Mit-

Der ursprüngliche Artikel wurde geändert, da nachträglich eine Open Access-Veröffentlichung gewünscht wurde. Diese Arbeit ist Teil des Leitthemas „Psychotherapie mit Kindern und Jugendlichen“.

D. Fiedler $\cdot$ K. Weitkamp

Department Gesundheit, MSH Medical School Hamburg, Hamburg, Deutschland

G. Romer

Klinik für Kinder- und Jugendpsychiatrie, -psychosomatik und -psychotherapie, Universitätsklinikum Münster,

Münster, Deutschland

S. Wiegand-Grefe $(\bowtie)$

Klinik für Kinder- und Jugendpsychiatrie, -psychosomatik und -psychotherapie, Universitätsklinikum

Hamburg-Eppendorf, Hamburg, Deutschland

s.wiegand-grefe@uke.de tel erreicht. Die Veränderung der Beeinträchtigungsschwere zwischen den beiden Erhebungszeitpunkten vor und nach der Therapie war statistisch bedeutsam mit großer Effektgröße. Zwischen der Verbesserung der Beeinträchtigungsschwere und der Zielerreichung zeigte sich ein signifikanter Zusammenhang mittlerer Effektgröße.

Schlussfolgerung Die Ergebnisse legen nahe, dass analytische Psychotherapie im Hinblick auf die Beeinträchtigungsschwere und Therapieziele eine wirksame Behandlungsmethode für Kinder und Jugendliche mit externalisierender Symptomatik sein kann.

Schlüsselwörter Externalisierende Störungen · Kinder · Jugendliche $\cdot$ Beeinträchtigungsschwere $\cdot$ Therapieziele $\cdot$ Analytische Psychotherapie

Changes of the impairment severity and therapeutic goal achievement through analytic psychotherapy in children and adolescents suffering from externalizing symptoms

\section{Summary}

Aim We analysed changes of impairment severity and therapeutic goal achievement within analytic psychotherapy in children and adolescents with externalizing symptoms.

Methods The data were part of a controlled intervention study with prospective study design, whereas in this article impairment severity and goal achievement were analysed by a within-group design only. 60 children and adolescents aged 4 to 17 years $(M=11.25$; $\mathrm{SD}=3.91 ; 22$ male) with high levels of externalizing symptoms in outpatient psychotherapy participated in the study. Impairment severity was rated by the therapist with the Impairment Score for Children and Adolescents (IS-CA). Goal achievement was reported by the therapists as well. 
Results The change of the impairment severity across the therapy interval was statistically significant with a large effect size. Therapists reported mostly interpersonal goals. Overall, goals were reported as achieved at the end of therapy. There was a significant correlation between the change of impairment severity and goal achievement with a medium effect size.

Conclusion The results suggest that analytic psychotherapy may be an effective treatment regarding impairment severity and therapeutic goal achievement for children and adolescents with externalizing symptoms.

Keywords Externalizing disorders - Children · Adolescents - Impairment severity - Therapeutic goals · Analytic psychotherapy

\section{Einleitung und Hintergrund}

Externalisierende Störungen wie Hyperkinetische Störungen (Döpfner 2011) und Störungen des Sozialverhaltens (Blanz 2011) sind im Kindes- und Jugendalter weit verbreitet. In den Leitlinien der Deutschen Gesellschaft für Kinder- und Jugendpsychiatrie und Psychotherapie (DGKJP 2007) zur Behandlung von externalisierenden Störungen werden kognitive und medikamentöse Interventionen empfohlen. Bei Vorliegen komorbider Störungen werden zusätzlich tiefenpsychologische Therapieverfahren nahegelegt (Gilmore 2000; Hopf 2007). Die Erforschung der analytischen Psychotherapie in diesem Bereich ist notwendig, da es an randomisierten, gut kontrollierten Studien in diesem Bereich mangelt (Kronmüller und Hartmann 1997). Insbesondere auch im Vergleich zur Verhaltenstherapie bzw. zu Elterntrainings, die bereits viele Belege vorweisen können (Fonagy et al. 2015), gibt es einen dringenden Forschungsbedarf. Zwar zeigen aktuelle vergleichende Therapiestudien unter Berücksichtigung gesundheitsökonomischer Parameter, dass die entstehenden Kosten der analytischen Langzeitpsychotherapie bei externalisierenden Störungen unter Einbeziehung der zusätzlich erforderlichen, symptombezogenen Interventionen wie (teil-) stationäre Aufenthalte geringer sind als die der medikamentengestützten Verhaltenstherapie (Laezer et al. 2015) und somit ökonomische Vorteile vorliegen, aber auch diese Studie ist angesichts der Studienlage und des Designs eher als vorläufiger Wirksamkeitsnachweis zu betrachten. Aus psychodynamischer Sicht werden derartige Störungsbilder nicht selten durch innere Konflikte und frühkindliche Entwicklungs-, Bindungs- und Beziehungsstörungen sowie infantile Traumata ausgelöst und aufrechterhalten, die in der psychoanalytischen Behandlung thematisiert werden können (Hopf 2007; Häussler 2002). Hierbei kommen der psychoanalytischen Behandlung die umfassende Berücksichtigung neurobiologischer, biographischer und gesellschaftlicher Faktoren zugute (Fischmann et al. 2007). So konnten Fonagy und Target (1994) die Wirksam- keit von analytischer Behandlung bei Kindern mit externalisierenden Störungen belegen, wobei der Effekt bei Kindern mit Störungen mit oppositionell-aufsässigem Verhalten größer war (56\% Verbesserungsrate) als bei denen mit hyperkinetischen Störungen (36\% Verbesserungsrate) und Störungen des Sozialverhaltens (23\% Verbesserungsrate).

Für die analytische Psychotherapie ist die Betrachtung der Symptomreduktion zwar wichtig, aber nur eingeschränkt bedeutsam (Ehlers und Holder 2009), da bei einer Langzeittherapie zudem eher eine Optimierung des allgemeinen Funktionsniveaus und speziell bei der analytischen Langzeittherapie die Aufarbeitung der eigenen Lebensgeschichte über die Symptomreduktion hinaus angestrebt wird (Brockmann et al. 2003). Daher werden in der vorliegenden Arbeit alternative Kriterien für Therapieerfolg fokussiert: die Formulierung von Therapiezielen, das Erreichen der Ziele im Therapieverlauf sowie die Beeinträchtigungsschwere. Einige Belege sprechen bereits für eine signifikante Verbesserung der Beeinträchtigungsschwere, auch in der analytischen Psychotherapie bei Kindern (Fahrig et al. 1996b; Dührssen 1964; Fonagy und Target 1994; Stefini et al. 2008). Hierbei sind die Effektstärken vergleichbar mit denen von kognitivbehavioralen Verfahren (Kronmüller und Hartmann 1997; Fahrig et al. 1996b). Unterschiede äußern sich eher inhaltlich, wobei Verhaltenstherapeuten Bewältigungskompetenzen und Prognosen positiver bewerten als tiefenpsychologische Psychotherapeuten, die vermehrt auf frühkindliche Belastungen achten (Faller et al. 2000). Winkelmann und Kollegen konnten in der analytischen Psychotherapie bei Kindern und Jugendlichen sowohl für Kurzzeitpsychotherapie (2005), aber auch für Langzeittherapie (2000) eine signifikant bessere Beeinträchtigungsschwere feststellen. In der Kurzzeittherapie äußerte sich dies mit einer Effektstärke von $E S=0,6$ (Cohen's $d$ ) im Vergleich von Therapie- und Wartekontrollgruppe und in der Langzeittherapie mit einer Effektstärke von $d=1,57$ für die Gesamtwerte innerhalb der Therapiegruppe. In der Studie von Fahrig et al. (1996b) zeigte sich mit $d=1,58$ eine ähnlich hohe Effektstärke. Die Ergebnisse sprechen für stabile Therapieerfolge, welche sich teilweise nach der Therapie sogar noch verbessern. Dies ist nicht ungewöhnlich für psychodynamische Therapien (Midgley und Kennedy 2011), in welchen ein langsamer, aber beständiger Wirksamkeitsprozess, auch noch nach der Therapie beobachtet wurde (Trowell et al. 2003, 2007). Fonagy und Target (1994) liefern zudem Belege für ein besseres Therapieergebnis bei höherer Therapiefrequenz und längerer Therapiedauer.

Das Erreichen von Therapiezielen stellt einen Prädiktor für den Therapieerfolg dar (Kazdin 1999; Kordy und Hannover 1999). Unter Zielen versteht man „gerichtete Veränderungen des Verhaltens und Erlebens, die ein Patient zu Beginn der Psychotherapie mit dem Therapeut vereinbart und mit Hilfe der Therapie zu 
erreichen versucht" (Grosse Holtforth 2001, S. 242). Bislang sind Ziele eher selten Gegenstand der empirischen Forschung (Gerhards 2008) insbesondere im Bereich der Kinder- und Jugendtherapie (Winter et al. 2005). Zur wissenschaftlichen Betrachtung der Therapieziele stehen für den Erwachsenenbereich Klassifikationssysteme zur Verfügung. Etabliert ist hier z.B. das Berner Inventar für Therapieziele (BIT-T; Grosse Holtforth und Grawe 2002). Eingeordnet ins BIT-T beobachtete Gerhards (2008) folgende Verteilung der Therapieziele bei Erwachsenen: 39,2 \% Selbstentwicklung, wobei tiefenpsychologische Psychotherapeuten diese Angabe mit 66,5\% wesentlich häufiger machten als verhaltenstherapeutische Psychotherapeuten $(23,4 \%), 35 \%$ Problem- und Symptombewältigung, 10,7\% interpersonelle Ziele, 5,9\% Wohlbefinden/,better functioning“, 1,0\% Orientierung und 8,3\% in der Restkategorie. Dass Verhaltenstherapeuten vermehrt Therapieziele hinsichtlich der Symptomatik äußern, lässt sich auch bei Schulte-Bahrenberg (1990) beobachten $(65,9 \%)$, wohingegen nur $18,4 \%$ den intrapersonellen Bereich betreffen. Kinder und Jugendlichen benannten mit $45 \%$ wiederum gehäuft intrapsychische Therapieziele (Winter et al. 2005). Das BIT zur Kategorisierung von Therapiezielen wurde für den Erwachsenenbereich entwickelt und soll im Rahmen dieser Studie explorativ auf den Kinder- und Jugendbereich angewendet werden.

Hinsichtlich der Therapieziele formulierten tiefenpsychologische Psychotherapeuten zum großen Teil Ziele im Bereich Selbstentwicklung. Es wird angenommen, dass dies bei analytischen Kinder- und Jugendlichenpsychotherapeuten vergleichbar ist. In diesem Zusammenhang wenig untersucht ist die Frage, ob die anfangs formulierten Therapieziele auch erreicht werden. Da reduzierte Beeinträchtigungsschwere und Zielerreichung jeweils mit Therapieerfolg zusammenhängen, liegt es nahe, dass auch die beiden Parameter miteinander korrelieren. Unter Berücksichtigung des derzeitigen Forschungsstandes ergeben sich folgende Hypothesen:

Hyp. 1 Die Beeinträchtigungsschwere verringert sich signifikant vom ersten zum zweiten Erhebungszeitpunkt (Therapiebeginn bis Therapieende).

Hyp. 2 Die Verbesserung der Beeinträchtigungsschwere über die Zeit ist bei zwei wöchentlichen Therapiesitzungen signifikant größer als bei einer wöchentlichen Therapiesitzung.

Hyp. 3 Die Verbesserung der Beeinträchtigungsschwere über die Zeit steigt mit längerer Therapiedauer.

Hyp. 4 Die Therapeuten äußern am häufigsten Zielangaben in der Oberkategorie Selbstentwicklung.
Hyp. 5 Die Therapeuten schätzen die Therapieziele im Anschluss an die Behandlung im Durchschnitt als erreicht ein.

Hyp. 6 Es besteht ein signifikanter Zusammenhang zwischen der Reduktion der Beeinträchtigungsschwere zwischen den Erhebungszeitpunkten und der eingeschätzten Zielerreichung.

\section{Methodik}

\section{Vorgehensweise}

Die hier berichtete Studie war Teil einer größeren Studie, der Hamburger Wirksamkeitsstudie zur psychoanalytischen Behandlung von Kindern und Jugendlichen mit Ängsten, Depressionen und externalisierenden Störungen (Wiegand-Grefe et al. 2011). Bei dem Untersuchungsdesign handelte es sich um eine kontrollierte Feldstudie mit prospektivem Studiendesign mit dem Ziel, die Wirksamkeit analytischer Kinder- und Jugendpsychotherapie im natürlichen Versorgungskontext bei Angst-, depressiven und externalisierenden Störungen zu prüfen. Das Augenmerk richtete sich in der hier berichteten Teilstudie auf den Verlauf der Beeinträchtigungsschwere, die Erreichung der Therapieziele sowie den Zusammenhang dieser beiden Variablen in der Gruppe der Patient/innen mit externalisierender Symptomatik. Da die Beeinträchtigungsschwere und die Therapiezielerreichung nicht bei Kontrollkindern erfasst wurden, war kein Gruppenvergleich möglich. In Kooperation mit niedergelassenen analytischen Kinder- und Jugendlichen-Psychotherapeuten erhielten die teilnehmenden Kinder und Jugendlichen eine individuell angepasste ambulante analytische Psychotherapie, welche vorwiegend mit den Kindern und teilweise in gemeinsamen Sitzungen mit den Eltern stattfand (Verhältnis 4:1). Als naturalistische Praxisstudie aus dem Bereich der Versorgungsforschung entsprach die Therapiedauer den typischen Bedingungen, welche durch die deutsche Krankenversicherung auf 150 Sitzungen bei Kindern und 180 Sitzungen bei Jugendlichen begrenzt ist. Die Studie wurde von der Ethikkommission der Ärztekammer Hamburg genehmigt.

Die Rekrutierung der Versuchspersonen fand zwischen September 2007 und Oktober 2012 statt. Die Versuchspersonen meldeten sich selbst in den niedergelassenen Praxen für eine Psychotherapie an, wo sie bzw. deren Eltern um Teilnahme gebeten wurden. Sie erhielten vom Therapeuten ein Informationsschreiben über die Studie und wurden um ihr schriftliches Einverständnis gebeten. Anschließend erhielten die Familien und die Therapeuten, wie auch bei späteren Erhebungen, aus der Studienzentrale der Klinik für Kinder- und Jugendlichen-Psychiatrie und Psychotherapie einen durch Codierung anonymisierten Fragebogen sowie weiterführende Instruktionen per Post, 
Tab. 1 Deskriptive Stichprobenbeschreibung

\begin{tabular}{|c|c|c|c|c|c|c|}
\hline & & $N$ & Anteil in \% & Range & $M$ & SD \\
\hline \multirow[t]{2}{*}{ Geschlecht } & Männlich & 22 & 36,7 & & & \\
\hline & Weiblich & 38 & 63,3 & & & \\
\hline Alter zu Therapiebeginn & & & & 4-17 Jahre & 11,25 & 3,913 \\
\hline \multirow[t]{2}{*}{ Therapiedauer } & Kurzzeittherapie & 11 & 18,3 & 7-25 Sitzungen & 24,45 & 1,81 \\
\hline & Langzeittherapie & 43 & 71,7 & 30-172 Sitzungen & 110,09 & 48,03 \\
\hline \multirow[t]{3}{*}{ Sitzungen/Woche } & 1 Stunde & 29 & 48,3 & & & \\
\hline & 2 Stunden & 28 & 46,7 & & & \\
\hline & Nicht bekannt & 3 & 5,0 & & & \\
\hline \multirow{6}{*}{$\begin{array}{l}\text { Störungen zu Therapiebeginn } \\
\text { (K-SADS bei } n=45 \text { ) }\end{array}$} & Externalisierende Störung & 25 & 54,3 & & & \\
\hline & Affektive Störung & 15 & 32,6 & & & \\
\hline & Angststörung & 17 & 37,0 & & & \\
\hline & $\begin{array}{l}\text { Anpassungs- und Belastungsstö- } \\
\text { rungen }\end{array}$ & 13 & 28,3 & & & \\
\hline & $\begin{array}{l}\text { Fütter-, Ess-, Tic- oder Ausschei- } \\
\text { dungs-störungen }\end{array}$ & 14 & 30,4 & & & \\
\hline & Komorbidität & 26 & 56,5 & & & \\
\hline
\end{tabular}

der auch ein frankierter Briefumschlag für die Rücksendung beinhaltete. Bei ausbleibender Rückmeldung erhielten die Familien nach zwei und vier Wochen ein Erinnerungsschreiben. Die Therapeuten erhielten eine Aufwandsentschädigung von 30 Euro für jeden teilnehmenden Patienten und auch die Patienten erhielten eine Aufwandsentschädigung für das Ausfüllen der Fragebögen. Die Patienten und die teilnehmenden Angehörigen erhielten jeweils 50 Euro für ihre Teilnahme. Vor Beginn der Therapie wurde ein halbstrukturiertes diagnostisches Interview mit den Probanden bzw. deren Eltern durchgeführt.

\section{Stichprobe}

In die Studie eingeschlossen wurden Kinder und Jugendliche zwischen vier und 17 Jahren mit auffälliger externalisierender Symptomatik im Selbstoder Elternurteil (Werte über dem Cut-off in der externalisierenden Syndromskala der Child Behaviour Checklist/Youth Self Report; Arbeitsgruppe Deutsche Child Behavior Checklist 1998a, b). Ausschlusskriterien waren eine psychotische Symptomatik, akute Suizidalität oder mangelnde deutsche Sprachkenntnisse. Die Gruppe der Patient/-innen mit externalisierenden Störungen bestand aus 60 Kindern und Jugendlichen, von denen 22 männlich $(36,7 \%)$ waren (s. Tab. 1). Der Altersdurchschnitt lag $\mathrm{zu}$ Therapiebeginn bei 11,25 Jahren $(S D=3,391)$. Die Teilnehmer erhielten eine $(48,3 \%, n=29)$ oder zwei $(46,7 \%, n=28)$ Stunden Psychotherapie pro Woche (nicht bekannt $5 \%$, $n=3)$. Im Mittel waren die Teilnehmer insgesamt 93,18 (SD = 60,16) Stunden in Therapie, wobei 12 Personen (20\%) eine Kurzzeittherapie im Umfang von bis zu 25 Stunden erhielten und 48 Personen eine Langzeittherapie $(80 \%)$. Neben einer externalisierenden Symptomatik litten 26 Probanden $(76,5 \%)$ unter einer komorbiden Störung. Am häufigsten traten affektive
Störungen (32,4\%), Anpassungs- und Belastungsstörungen $(29,4 \%)$ sowie Angststörungen (23,5\%) auf, aber auch Fütter-, Ess-, Tic- oder Ausscheidungsstörungen (s. Tab. 1).

\section{Erhebungsinstrumente}

Die angegebenen Erhebungsinstrumente waren Teil einer umfassenden Fragebogenbatterie der Gesamtstudie.

Die Beeinträchtigungsschwere wurde mittels des Beeinträchtigungsschwere-Score für Kinder und Jugendliche (BSS-K) von Fahrig et al. (1996) erhoben, ein Instrument zur Einschätzung des Schweregrades psychischer Störungen sowie daraus resultierende Beeinträchtigungen des allgemeinen Funktionsniveaus bei Kindern aus Therapeutenperspektive. Der Fragebogen ist ein adaptiertes und evaluiertes Verfahren des BSS von Schepank (1995) aus dem Erwachsenenbereich mit einer Interraterreliabilität zwischen $K=$ 0,73 und $K=0,84$ für die Einzelskalen und bei $K=0,80$ für den Gesamtwert sowie Retest-Reliabilität-Koeffizienten zwischen $r_{\mathrm{tt}}=0,73$ und $r_{\mathrm{tt}}=0$,86 für einzelne Skalen und $r_{\mathrm{tt}}=0,84$ für den Gesamtwert (Kronmüller et al. 2005). Dem Therapeuten stehen beim BSS-K (Fahrig et al. 1996a) vier Dimensionen zur Verfügung: Psychische Beeinträchtigung, Körperliche Beeinträchtigung, Sozial-kommunikative Beeinträchtigung mit Eltern, Geschwistern und Freunden und Beeinträchtigung im Leistungsbereich. Der Gesamtwert ergibt sich aus den einzelnen Dimensionen mit je fünf Stufen. Stufe 0 beschreibt laut Manual keine Hinweise auf eine psychisch bedingte Beeinträchtigung, Stufe 1 eine leichte, erkennbare Symptomatik mit geringer Einschränkung ohne aktive Anstrengung der Krankheitsbewältigung, Stufe 2 eine deutliche, beeinträchtigende Symptomatik, welche das Kind aber nicht weitgehend aus einzelnen Lebensbereichen 
ausschließt, Stufe 3 erhebliche Einschränkungen in bestimmten Lebensbereichen und Stufe 4 maximale klinische Behinderung durch extreme Intensität der Symptomatik. Mehrere geringere Beeinträchtigungen können addiert den gleichen Punktwert in einer Dimension erreichen wie eine starke Beeinträchtigung. Klinische Auffälligkeit ist ab einem Gesamtwert von 7 anzunehmen und eine bedeutsame Veränderung bei einer Verbesserung um mindestens 4 Punkte (Fahrig et al. 1996a).

Die Therapieziele wurden mit zwei ad hoc formulierten Items erfasst. Die Therapeuten wurden zu Therapiebeginn aufgefordert, in einem leeren Textfeld die Therapieziele aufzuschreiben, wobei die Anzahl nicht beschränkt wurde. Nach Beendigung der Therapie wurden die behandelnden Psychotherapeuten nach ihrer Einschätzung gefragt, inwiefern ihre Patienten die zu Beginn formulierten Ziele ihrer Ansicht nach erreicht haben. Hierfür stand ihnen eine fünfstufige Skala zur Verfügung, von 1 = überhaupt nicht, bis 5 = voll und ganz.

Diagnosestellung der psychischen Störungen wurde mit dem semistrukturierten Interview Schedule for Affective Disorders and Schizophrenia (K-SADS) von Delmo und Kollegen ermittelt (2001), welches die Diagnosen von ICD-10 und DSM-IV berücksichtigt. Das diagnostische Interview wurde durch speziell geschulte Mitarbeiter/-innen mit den Probanden bzw. deren Eltern durchgeführt.

Externalisierende Symptomatik wurde mit der Child Behaviour Checklist/Youth Self Report (CBCL/ YSR) (Arbeitsgruppe Deutsche Child Behavior Checklist 1998a, b) erfasst. Die CBCL/YSR besteht aus 118 Items zu spezifischen emotionalen und Verhaltensproblemen in Kindheit und Jugend. Es lassen sich aus den Subskalen auch eine internalisierende und eine externalisierende Syndromskala berechnen. Jedes Item steht für ein Problem und wird auf einer dreistufigen Skala von $0=$ nicht zutreffend bis $2=$ genau oder häufig zutreffend im Selbst- oder Elternurteil beantwortet. Reliabilität und Validität wurden vielfach belegt (Ebesutani et al. 2011).

\section{Auswertung}

Die Kategorisierung der Therapieziele erfolgte mit dem Berner Inventar der Therapieziele (BIT-T) von Grosse Holtforth und Grawe (2002). Hierbei handelt es sich um ein Instrument zur repräsentativen Darstellung von Therapiezielen im ambulanten Therapiebereich. Die Therapieziele werden nach inhaltlicher Ähnlichkeit gruppiert. Daraus ergeben sich drei Abstraktionsebenen, fünf Oberkategorien, 31 Kategorien, 50 Unterkategorien und die Restkategorien, wobei in dieser Untersuchung nur die Oberkategorien betrachtet wurden. Dabei handelt es sich um die Bereiche Problem- und Symptombewältigung (z. B. Lernen, besser mit meinen Stimmungsschwankungen umzugehen), Interpersonale Ziele (z. B. mit Kränkung,
Kritik umgehen können), Wohlsein/better functioning (z. B. gelassener sein), Orientierung (z. B. Entdecken eigener Interessen) und Selbstentwicklung (z. B. aus Ich-Zentriertheit herausfinden). In der vorliegenden Studie wurden die Therapieziele im Rahmen zweier Bachelorarbeiten von Kurock (2014) und Mäß (2014) anhand des Berner Inventar für Therapieziele (BITT) von Grosse Holtforth und Grawe (2002) mithilfe des Programmes MaxQDA 10 den Klassifikationskategorien zugeordnet. Es konnte jeweils mehr als eine Kategorie vergeben werden. Zwei Rater beurteilten die Therapieziele unabhängig voneinander. Die Übereinstimmung der Rater betrug insgesamt 88,8\%. Bei Differenzen wurde eine dritte unabhängige Person und für ein besseres Verständnis analytischer Formulierungen zudem eine analytische Kinder- und Jugendlichenpsychotherapeutin hinzugezogen und im Konsens geklärt. Die weiteren Auswertungen basierten auf der finalen Kategorisierung.

Statistische Auswertung: In den vorliegenden Berechnungen wurden nur die Fälle berücksichtigt, zu denen vollständige Daten vorlagen (Complete Cases). Eine Intent-to-treat Analyse, mit einer Ersetzung fehlender Werte durch einfaches Last-observation-carried-forward, führte zu vergleichbaren Ergebnissen. In der Hauptpublikation (Weitkamp et al. eingereicht) wurde zusätzlich eine verzerrungsfreie Ersetzung mittels Multiple Imputation durchgeführt, die im Hinblick auf die externalisierende Symptomatik ebenfalls vergleichbare Ergebnisse lieferte. Für die verwendeten inferenzstatistischen Tests der vorliegenden Studie wurden die teststatistischen Voraussetzungen wie folgt geprüft: Das Vorliegen von Normalverteilung wurde mit dem Kolmogorov-Smirnov-Anpassungstest (K-S bei einer Stichprobe) getestet. Die absolute Differenz für den BSS-K zu Therapiebeginn beträgt Prüfgröße der Differenz $(P G)_{\mathrm{n} 52}=0,165$ und liegt unter der $\mathrm{K}-\mathrm{S}$-Verteilung (kritischer Wert $_{\mathrm{n} 52}=0,188$ ) und auch jene für den BSS-K zu Therapieende liegt mit $P G_{\mathrm{n} 45}=$ 0,148 unter der K-S-Verteilung (kritischer Wert $_{\mathrm{n} 45}=$ 0,202). Somit kann eine Normalverteilung angenommen werden. Die Varianzhomogenität ist aufgrund wiederholter Messungen derselben Stichprobe sichergestellt. Es gab keinerlei Verletzungen der Voraussetzungen für die Durchführung der statistischen Verfahren. Die statistische Auswertung der Daten erfolgt mittels des Datenverarbeitungsprogrammes IBM SPSS Statistics 21. Das Signifikanzniveau wurde nach gängigen Kriterien bei $\alpha=0,05$ festgesetzt. Die Effektstärken wurden nach Cohen (1969) beurteilt.

Die Veränderung der Beeinträchtigungsschwere über die Zeit wurde mit einer Varianzanalyse mit Messwiederholung berechnet. Hierbei wurde der Gesamtwert herangezogen, ergänzend wurden die Einzelskalen berücksichtigt. Die Therapiefrequenz (ein bzw. zwei Stunden pro Woche) sowie die Anzahl der Stunden pro Woche wurden als Kovariate mit aufgenommen. Wie viele Patienten eine klinisch signifikante und reliable Veränderung aufwiesen, wurde im 
Tab. 2 Varianzanalyse mit Messwiederholung der BSS-K-Gesamtwerte

\begin{tabular}{|c|c|c|c|c|c|c|c|c|}
\hline & \multicolumn{2}{|c|}{ Therapiebeginn } & \multicolumn{2}{|c|}{ Therapieende } & \multirow[b]{2}{*}{ df } & \multirow[t]{2}{*}{$F$} & \multirow[t]{2}{*}{$p$} & \multirow[t]{2}{*}{ Partielles $\eta^{2}$} \\
\hline & $M$ & SD & $M$ & SD & & & & \\
\hline Gesamtwert & 10,29 & 2,79 & 5,78 & 3,87 & $1 / 38$ & 6,929 & 0,011 & 0,126 \\
\hline Psychische Beeinträchtigung & 2,25 & 0,65 & 1,07 & 0,93 & $1 / 43$ & 56,773 & $<0,001$ & 0,569 \\
\hline Körperliche Beeinträchtigung & 0,69 & 0,85 & 0,20 & 0,46 & $1 / 44$ & 13,427 & 0,001 & 0,234 \\
\hline Sozial-kommunikative B./Eltern & 2,33 & 1,02 & 1,29 & 1,04 & $1 / 44$ & 38,631 & $<0,001$ & 0,468 \\
\hline Sozial-kommunikative B./Geschwister & 1,73 & 1,18 & 0,88 & 0,93 & $1 / 32$ & 16,440 & $<0,001$ & 0,339 \\
\hline Sozial-kommunikative B./Freunde & 2,00 & 0,99 & 0,82 & 0,93 & $1 / 39$ & 60,203 & $<0,001$ & 0,607 \\
\hline Beeinträchtigung im Leistungsbereich & 1,60 & 1,23 & 0,79 & 0,98 & $1 / 41$ & 21,505 & $<0,001$ & 0,344 \\
\hline
\end{tabular}

Abb. 1 Einschätzung der Therapeuten hinsichtlich der Zielerreichung

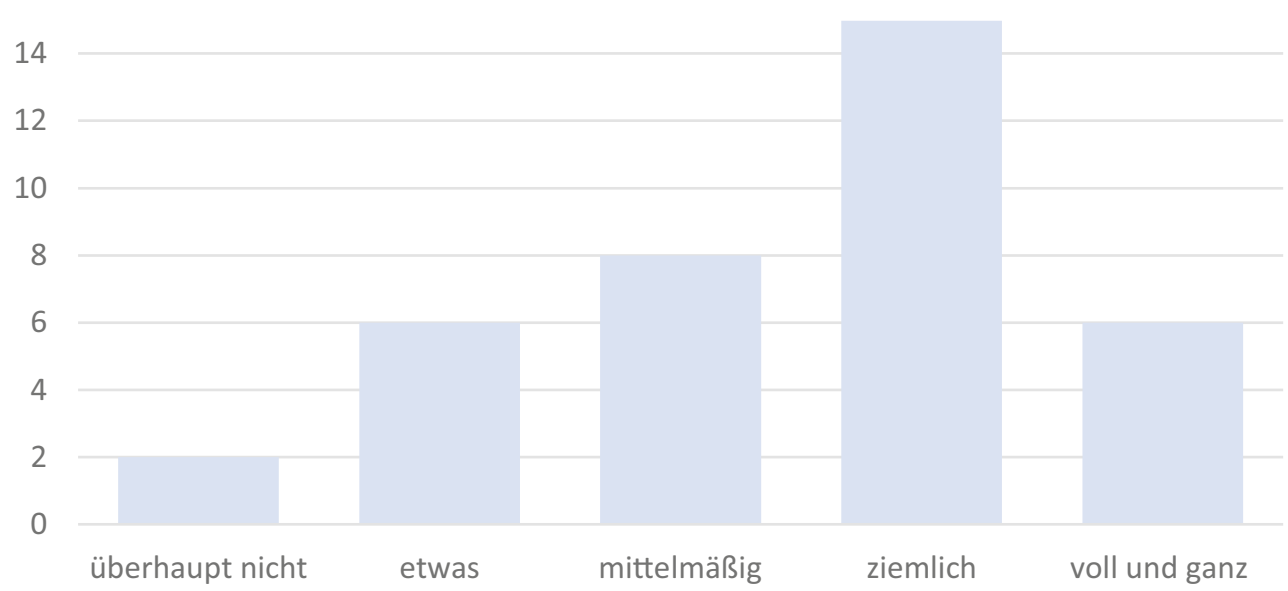

Sinne des Reliable Change Index (RCI) nach Jacobson und Truax (1991) berechnet. Für die Untersuchung der Therapiezielerreichung wurde die 5-stufige Antwortskala im Mittel bei 3 („mittelmäßig“) geteilt und dieser Wert als Schwelle festgelegt, sodass größere Werte als erreichtes Therapieziel eingeschätzt wurden. Ob der Mittelwert für die Zielerreichung über dem Kriteriumswert liegt, wurde mittels eines Einstichproben-tTests geprüft. In der fünften Fragestellung wurden die Veränderung der Beeinträchtigungsschwere und die Zielerreichung auf einen korrelativen Zusammenhang geprüft mit einer bivariaten Korrelation. Hierfür wurde die Differenz der BSS-K-Gesamtwerte der beiden Messzeitpunkte gebildet.

\section{Ergebnisse}

\section{Veränderung der Beeinträchtigungsschwere zu den Erhebungszeitpunkten}

Die Überprüfung der Veränderung der Beeinträchtigungsschwere über die Zeit (Hyp. 1) erfolgte mittels Varianzanalyse. Die Beeinträchtigungsschwere lag zu Therapiebeginn bei $M=10,29(S D=2,79)$ und $\mathrm{zu}$ Therapieende bei $M=5,78$ ( $S D=3,87$; s. Tab. 2). Verglichen mit dem ersten Erhebungszeitpunkt zu Therapiebeginn zeigte sich eine signifikante Verringerung der Beeinträchtigungsschwere zum zweiten Erhebungszeitpunkt/zu Therapieende $(F=6,929 ; p \leq 0,011)$ mit einer großen Effektstärke (Partielles $\eta^{2}=0,126$ ). Auch die Veränderung aller Einzelskalen war signifikant (s. Tab. 2). Hypothese 1 wird damit beibehalten. Die klinische Signifikanz und der RCI werden im Folgenden für die Complete Cases $(n=42)$ dargestellt: Eine klinisch signifikante Verbesserung zeigte sich für $59.5 \%$ der Patienten $(n=25)$. Bei $28.6 \%(n=12)$ ergab sich keine klinisch signifikante Verbesserung. Keiner der Patienten hatte sich vom unauffälligen in den auffälligen Bereich verschlechtert. Fünf Patienten (11,9\%) waren zu beiden Messzeitpunkten im BSS-K im unauffälligen Bereich. Bei $73.8 \%(n=31)$ der Patienten zeigte sich eine reliable Verbesserung der BSS-K-Werte, für $26,2 \%(n=11)$ zeigte sich keine reliable Veränderung, reliabel verschlechtert hat sich keiner der Patienten.

Zur Prüfung des Einflusses der Anzahl der Stunden pro Woche und der Therapiedauer (Hyp. 2 und 3) wurden die beiden Variablen als Kovariaten ins Modell mit aufgenommen. Weder die Frequenz der Therapiesitzungen $(F=0,844, p \leq 0,364)$ noch die Anzahl der Sitzungen pro Woche $(F=2,804, p \leq 0,102)$ waren signifikant.

\section{Ziele und eingeschätzte Zielerreichung}

Zur Prüfung der Hypothese 4 wurden die Häufigkeiten der Arten von Therapiezielen angeschaut. Die Therapeuten formulieren insgesamt 131 Ziele, die in Tab. 3 aufgelistet sind. Interpersonelle Ziele wurden mit 49 Angaben (37,40\%) am häufigsten formuliert. 
Tab. 3 Deskriptive Statistiken der Therapiezielangaben

\begin{tabular}{|l|l|l|}
\hline Interpersonelle Ziele & Anzahl der Therapiezielangaben & Anteil an der Gesamtzahl aller Ziele (\%) \\
\hline Selbstentwicklung & 49 & 37,40 \\
\hline Problem- und Symptombewältigung & 47 & 35,88 \\
\hline Orientierung & 22 & 16,79 \\
\hline Wohlsein/better functioning & 7 & 5,34 \\
\hline Restkategorie & 3 & 2,29 \\
\hline Keine Angabe & 3 & 2,29 \\
\hline Gesamt & 4 & 3,05 \\
\hline
\end{tabular}

Zielangaben in der Oberkategorie Selbstentwicklung wurde von den Therapeuten am zweithäufigsten geäußert. Die Hypothese 4 wurde damit nicht bestätigt.

Das von den Therapeuten $\mathrm{zu}$ Therapieende eingeschätzte Ausmaß der Zielerreichung ist in Abb. 1 dargestellt (Hyp. 5). Im Mittel lag die Zielerreichung bei $M=3,54(S D=1,05)$ signifikant über dem erwarteten Testwert von 3 ( $t=3,326 ; p \leq 0,002)$. Demnach schätzten die Therapeuten die Therapieziele im Anschluss an die Behandlung im Durchschnitt als erreicht ein. Die Hypothese 5 wurde somit beibehalten.

\section{Zusammenhang zwischen \\ Beeinträchtigungsschwere und Zielerreichung}

Mittels Korrelation wurde der Zusammenhang zwischen der Veränderung der Beeinträchtigungsschwere und der Zielerreichung geprüft (Hyp. 6). Die Differenz zwischen den Werten der Beeinträchtigungsschwere vor und nach der Therapie beträgt im Mittel $M=5,74$ und die deskriptive Einschätzung der Zielerreichung $M=3,54$ ( $S D=1,05)$. Die bivariate Korrelation nach Pearson ergab bei einseitiger Testung einen signifikanten Zusammenhang, $r=34(p \leq 0,001)$ in erwarteter Richtung. Der ermittelte Zusammenhang lag im Bereich mittlerer Effektgrößen. Eine größere Verbesserung der Beeinträchtigung ging mit einer höher eingeschätzten Zielerreichung einher.

\section{Diskussion}

In der vorliegenden Arbeit wurde im Therapeutenurteil die Veränderung der Beeinträchtigungsschwere sowie die Therapiezielerreichung durch analytische Psychotherapie bei Kindern und Jugendlichen mit externalisierender Symptomatik betrachtet. Es zeigte sich eine Reduktion der Beeinträchtigungsschwere zwischen den beiden Erhebungszeitpunkten. Zusätzlich gaben die Therapeuten im Durchschnitt an, die eingangs formulierten Therapieziele erreicht $\mathrm{zu}$ haben. Weder eine höhere Therapiefrequenz noch eine längere Therapiedauer zeigte in dieser Stichprobe einen Einfluss auf die Veränderungen der Beeinträchtigungsschwere. Die Reduktion der Beeinträchtigungsschwere hing signifikant mit der eingeschätzten Zielerreichung zusammen.
Hinsichtlich der verringerten Beeinträchtigungsschwere zeigten sich in Vorgängerstudien sowohl nach psychodynamischer Kurzzeitpsychotherapie als auch nach Langzeittherapie ähnliche Ergebnisse (Winkelmann et al. 2000, 2005; Fahrig et al. 1996b). Der verbesserte Gesamtwert kann für ein gutes Therapieergebnis sprechen. In allen Einzelskalen des BSS-K konnte im Einklang mit den Arbeiten von Fahrig et al. (1996b) und Winkelmann et al. (2000) eine signifikante Verbesserung festgestellt werden. Die Beeinträchtigungsschwere lag bei Therapiebeginn ähnlich hoch, was für vergleichbare Patientengruppen spricht. Auf den Einzelskalen lassen sich die größten Effekte in der psychischen Beeinträchtigung und der sozialkommunikativen Beeinträchtigung bzgl. der Eltern feststellen, wie auch bei Fahrig et al. (1996b) und bei Winkelmann et al. (2000), dort jedoch in umgekehrter Reihenfolge. Im Unterschied zu Fonagy und Target (1994) zeigten sich in der aktuellen Stichprobe keine Auswirkungen der Therapiedauer und -frequenz auf den Therapieerfolg, was ggf. an der hohen Komorbiditätsrate einhergehend mit einem hohen Anteil von Langzeittherapien liegen kann.

Die Zielerreichung wurde im Durchschnitt als erreicht eingeschätzt. Dabei umfassen interpersonelle Ziele den Großteil der Angaben, welche bei Gerhards (2008) und Schulte-Bahrenberg (1990) jeweils nur einen kleinen Anteil ausmachen. Jedoch berücksichtigen beide Studien nur Erwachsene. Die Studie von Schulte-Bahrenberg beinhaltete zudem nur internalisierende Störungen in einer verhaltenstherapeutischen Behandlung mit retrospektiver Erfassung, wohingegen bei Gerhards die psychodynamische Therapie untersucht wurde. Letzteres könnte möglicherweise den übereinstimmend hohen Anteil des zweithäufigsten genannten Zieles, der Selbstentwicklung, erklären. Überdies zeigte sich, dass Problemund Symptombewältigung nicht das vorrangige Ziel der Therapie war. $\mathrm{Zu}$ berücksichtigen ist, dass interpersonelle Probleme bei Kindern und Jugendlichen entwicklungsbedingt eine andere Rolle spielen als im Erwachsenenalter, da sie in den Lebenskontexten Familie, Schule und Freundschaften neue und prägende Erfahrungen machen. In Folgestudien wäre zu prüfen, ob sich die vorliegenden Ergebnisse replizieren lassen. Um eine bessere Vergleichbarkeit zu gewähr- 
leisten, könnte in zukünftigen Studien zum Beispiel die Anzahl der Therapiezielangaben festgelegt werden und die Therapieziele im Anschluss der Therapie einzeln auf ihre Erreichung beurteilt werden.

Die Einschätzung der Zielerreichung hing signifikant mit der Reduktion der Beeinträchtigungsschwere zusammen. Der Effekt lag im mittleren Bereich. Da sich die Beeinträchtigungsschwere im Verlauf der Therapie verbessert hat und somit als Mediator des Therapieerfolgs anzusehen ist, verwundert es wenig, dass mit einer hoch beurteilten Zielerreichung, welche als Korrelat des Therapieerfolgs $\mathrm{zu}$ sehen ist, auch eine verbesserte Beeinträchtigungsschwere einhergeht. Bisherige Befunde thematisieren schwerpunktmäßig einzelne Prädiktoren in Zusammenhang mit Therapieerfolg statt den Zusammenhang zweier Prädiktoren selbst, wobei zum Beispiel häufiger vorkommt, dass Symptomreduktion und reduzierte Beeinträchtigungsschwere gemeinsam auftreten (Winkelmann et al. 2005; Fahrig et al. 1996b).

\section{Einschränkungen der Studie}

Das naturalistische Setting ermöglichte eine unverzerrte Abbildung des aktuellen Versorgungskontextes, mit Zugangswegen, wie sie regulär stattfinden und einer Stichprobe mit hohem Anteil an komorbiden Störungen. Die hohe externe Validität der Studie geht jedoch mit den folgenden Limitationen einher, die bei der Interpretation der Ergebnisse zu berücksichtigen sind. Bei der Studie handelte es sich um ein Wartelisten-Kontrollgruppendesign, die hier berichtete Beeinträchtigungsschwere wurde jedoch in der Kontrollgruppe nicht erfasst und konnte somit nicht verglichen werden. Inwieweit die Stichprobe des Weiteren von der Gesamtheit der von externalisierenden Störungen betroffenen Kinder und Jugendlichen abweicht, kann hier nicht geklärt werden. Eine Verzerrung in Bezug auf die Gesamtpopulation ist nicht auszuschließen, da bestimmte Merkmale dazu führen, sich eher therapeutische Hilfe zu suchen (Steinhausen 2006). Des Weiteren unterschieden sich Dauer und Frequenz der Therapie. Die unterschiedliche Gestaltung der Therapie je nach Alter erschwert zudem einen direkten Vergleich innerhalb der Stichprobe. Das Alter wurde in der Hauptpublikation mit berücksichtigt. Dort zeigte sich jedoch kein signifikanter Einfluss (Weitkamp et al. eingereicht). Außerdem ist bei einer Längsschnittuntersuchung der Einfluss der Rahmenbedingungen nicht auszuschließen. Insbesondere bei der retrospektiven Erhebung am Ende der Therapie ist die Objektivität der Therapeuten fraglich, auch da die Beobachtungsmöglichkeiten auf die Sitzungen beschränkt sind, sodass die Therapeuten nur einen Ausschnitt des modifizierten Verhaltens und Erlebens einschätzen können. Die von den Therapeuten formulierten Therapieziele kommen den psychoanalytisch sinnvollen Erfolgskriterien nahe, waren aber teilweise nicht eindeutig in die Oberkategorien des
BIT-T (Grosse Holtforth und Grawe 2002) einordbar. Dadurch konnten spezifische inhaltliche Zielsetzungen weniger berücksichtigt werden, was jedoch als konservatives Vorgehen zu sehen ist. Die z. T. auftretenden Schwierigkeiten bei der Kategorisierung der Ziele, das für den Erwachsenenbereich konzipierte BIT-T auf die Lebenswelten von Kindern und Jugendlichen anzuwenden (z. B. Ziele in Bezug auf den Schulbesuch), könnte eine Erklärung für Abstriche in der Interraterreliabilität liefern. Fehlende Übereinstimmungen lagen zusätzlich möglicherweise an einer unterschiedlichen Tendenz, Therapieziele mehrfach zu kategorisieren, sodass inhaltlich ein Therapieziel in die gleiche Kategorie eingeordnet wurde, von einem Rater aber ggf. zusätzlich noch in eine andere Kategorie. Das BIT-T wurde in dieser Studie nach unserem Kenntnisstand erstmalig explorativ im Kinder- und Jugendbereich angewendet und diese Erfahrungen legen nahe, dass in einigen inhaltlichen Bereichen eine weitere Adaption wichtig wäre, wenn das BIT zukünftig in der Kinder- und Jugendpsychotherapieforschung weitergehende Verwendung finden sollte. Zukünftige Forschungsprojekte sollten daher die Entwicklung eines auf die analytische Therapieschule und auf Kinder und Jugendliche zugeschnittenes Kategoriensystems in Erwägung ziehen.

\section{Fazit}

Die Untersuchung des Therapieerfolgs nach analytischer Psychotherapie bei Kindern und Jugendlichen mit externalisierender Symptomatik in der Beurteilung der Therapeuten zeigte eine statistisch bedeutsame Reduktion der Beeinträchtigungsschwere. Die zu Beginn formulierten Therapieziele, die vor allem im Bereich der interpersonellen Ziele und der Selbstentwicklung genannt wurden, wurden im Mittel erreicht. Darüber hinaus besteht ein Zusammenhang zwischen der Reduktion der Beeinträchtigungsschwere und Erreichung der Therapieziele. In zukünftigen Studien sollten sowohl Beeinträchtigungsschwere und Zielerreichung als auch andere für die analytische Psychotherapie geeignete Prädiktoren für Therapieerfolg als Ergänzung zur Erfassung der Symptomreduktion überprüft werden.

Danksagung Wir danken den teilnehmenden Therapeut*innen und Familien für ihre Unterstützung. Weiterer Dank gilt Vivian Kurock und Mareike Mäß, die im Rahmen ihrer Psychologie Bachelorarbeiten die Zielkategorisierungen erarbeitet haben.

Förderung Die Studie wurde gefördert von der Vereinigung analytischer Kinder- und Jugendlichen Therapeuten (VAKJP e. V.). Die VAKJP hatte keinen Einfluss auf die Durchführung und Auswertung der Studie. 


\section{Einhaltung ethischer Richtlinien}

Interessenkonflikt D. Fiedler, K. Weitkamp, G. Romer und S. Wiegand-Grefe geben an, dass kein Interessenkonflikt besteht.

Ethische Standards Alle beschriebenen Untersuchungen am Menschen wurden mit Zustimmung der zuständigen EthikKommission, im Einklang mit nationalem Recht sowie gemäß der Deklaration von Helsinki von 1975 (in der aktuellen, überarbeiteten Fassung) durchgeführt. Von allen beteiligten Patienten liegt eine Einverständniserklärung vor.

Open Access Dieser Artikel wird unter der Creative Commons Namensnennung 4.0 International Lizenz (http:// creativecommons.org/licenses/by/4.0/deed.de) veröffentlicht, welche die Nutzung, Vervielfältigung, Bearbeitung, Verbreitung und Wiedergabe in jeglichem Medium und Format erlaubt, sofern Sie den/die ursprünglichen Autor(en) und die Quelle ordnungsgemäß nennen, einen Link zur Creative Commons Lizenz beifügen und angeben, ob Änderungen vorgenommen wurden.

\section{Literatur}

Arbeitsgruppe Deutsche Child Behavior Checklist (1998a). Handbuch: Fragebogen für Jugendliche - Deutsche Bearbeitung des Youth Self Report (YSR) der Child Behavior Checklist. Einführung und Anleitung zur Handauswertung. Köln: Arbeitsgruppe Kinder-, Jugend-, und Familiendiagnostik.

Arbeitsgruppe Deutsche Child Behavior Checklist (1998b). Elternfragebogen über das Verhalten von Kindern und Jugendlichen - Deutsche Bearbeitung der Child Behavior Checklist (2. Aufl.). S. CBCL/4-18). Köln: Arbeitsgruppe Kinder-, Jugend- und Familiendiagnostik (KJFD).

Blanz, B. (2011). Störungen des Sozialverhaltens und Jugenddelinquenz. In G. Esser (Hrsg.), Lehrbuch der klinischen Psychologie und Psychotherapie bei Kindern und Jugendlichen (S. 227-239). Stuttgart:Georg Thieme.

Brockmann, J., Schlüter, T., \& Eckert, J. (2003). Therapieziele, Zieländerungen und Zielerreichung im Verlauf psychoanalytisch orientierter und verhaltenstherapeutischer Langzeittherapie: Eine vergleichende Untersuchung aus der Praxis niedergelassener Psychotherapeuten. Psychotherapeutisch Psychosomatik Medizinische Psychologie, $53,163-170$.

Cohen, J. (1969). Statistical power analysis for the behavioral sciences. Hillsdale: Lawrence Erlbaum Associates.

Delmo, C., Weiffenbach, O., Gabriel, M., \& Poustka, F. (2001). Diagnostisches Interview. Kiddie-SADS Present and Lifetime Version ( $K-S A D S-P L)$. Frankfurt am Main: Klinik für Psychiatrie und Psychotherapie des Kindes- und Jugendalters.

Deutsche Gesellschaft für Kinder- und Jugendpsychiatrie und Psychotherapieu. a. (2007). Leitlinien zur Diagnostik und Therapie von psychischen Störungen im Säuglings-, Kindes- und Jugendalter (3. Aufl.). Köln: Deutscher Ärzte Verlag.

Döpfner, M. (2011). Hyperkinetische Störungen. In G. Esser (Hrsg.), Lehrbuch der klinischen Psychologie und Psychotherapie bei Kindern und Jugendlichen (S. 202-226). Stuttgart: Georg Thieme.

Dührssen, A. (1964). Katamnestische Untersuchung bei 150 Kindern und Jugendlichen nach analytischer Psychotherapie. Praxis der Kinderpsychologie und Kinderpsychiatrie, 7,241-255.
Ebesutani, C., Bernstein, A., Martinez, J.I., Chorpita, B. F., \& Weisz, J.R. (2011). The Youth Self Report: Applicability and validity across younger and older youths. Journal of Clinical Child and Adolescent Psychology, 40,338-346.

Ehlers, W., \& Holder, A. (2009). Psychoanalytische Verfahren. Basiswissen Psychoanalyse. Stuttgart: Klett-Cotta.

Fahrig, H., Hartmann, M., Kronmüller, K.-T. (1996a). Beeinträchtigungsschwere-Score für Kinder und Jugendliche. Rating-Manual. Unveröffentlichtes Manuskript.

Fahrig, H., Kronmüller, K.-T., Hartmann, M., \& Rudolf, G. (1996b). Therapieerfolg analytischer Psychotherapie bei Kindern und Jugendlichen: Die Heidelberger Studie zur Analytischen Kinder- und Jugendlichen-Psychotherapie. Zeitschrift für Psychosomatische Medizin und Psychoanalyse, 42, 375-395.

Faller, H., Wagner, R.F., \& Weiß, H. (2000). Therapeutische Orientierung und Wahrnehmung des Patienten bei der Erstuntersuchung-Ein empirischer Vergleich von tiefenpsychologisch/analytisch und kognitiv-verhaltenstherapeutisch orientierten Therapeuten. Psychotherapeut, 45, 108-115.

Fischmann, T., Leuzinger-Bohleber, M., \& Staufenberg, A. (2007). ADHS - Indikation für psychoanalytische Behandlungen? Einige klinische, konzeptuelle und empirische Überlegungen ausgehend von der Frankfurter Präventionsstudie. Praxis der Kinderpsychologie und Kinderpsychiatrie, 56, 356-385.

Fonagy, P., Cottrell, D., Phillips, J., Bevington, D., Glaser, D., \& Allison, E. (2015). What works for whom? A critical review of treatments for children and adolescents. NewYork: Guilford Publications.

Fonagy, P., \& Target, M. (1994). The efficacy of psychoanalysis for children with disruptive disorders. Journal of the American Academy of Child and Adolescent Psychiatry, 33, 45-55.

Gerhards, C. (2008). Therapieziele und Therapieerfolg. Diplomarbeit: Universität Osnabrück.

Gilmore, K. (2000). A psychoanalytic perspective on attention-deficit/hyperactivity disorder. Journal of the American Psychoanalytic Association, 48, 1259-1293.

Grosse Holtforth, M. (2001). Was möchten Patienten in ihrer Therapie erreichen? - Die Erfassung von Therapiezielen mit dem Berner Inventar für Therapieziele (BIT). Verhaltenstherapie und psychosoziale Praxis, 33, 241-258.

Grosse Holtforth, M., \& Grawe, K. (2002). Bern inventory of treatment goals: part 1. development and first application of a taxonomy of treatment goal themes. Psychotherapy Research, 12,79-99.

Häussler, G. (2002). Attention deficit and hyperkinetic syndrome (ADHS) from the psychoanalytic viewpoint. Praxis der Kinderpsychologie und Kinderpsychiatrie, 51, 454-465.

Hopf, H. (2007). Between empathy and setting limits-reflections on psychodynamics and treatment techniques in psychoanalytic therapies for hyperkinetic disorders. Praxis der Kinderpsychologie und Kinderpsychiatrie, 56, 333-355.

Jacobson, N.S., \& Truax, P. (1991). Clinical-significance - a statistical approach to defining meaningful change in psychotherapy-research. Journal of Consulting and Clinical Psychology, 59, 12-19.

Kazdin, A.E. (1999). The meanings and measurement of clinical significance. Journal of Consulting and Clinical Psychology, 67,332-339.

Kordy, H., \& Hannover, W. (1999). Zur Evaluation psychotherapeutischer Behandlungen anhand individueller Thera- 
pieziele. In H. Ambühl, \& B. Strauß (Hrsg.), Therapieziele (S.75-90). Göttingen: Hogrefe.

Kronmüller, K.-T., \& Hartmann, M. (1997). Psychoanalytische Therapieforschung bei Kindern und Jugendlichen. In $\mathrm{H}$. Mandl (Hrsg.), Bericht über den 40. Kongress der Deutschen Gesellschaft für Psychologie. Göttingen: Hogrefe.

Kronmüller, K.-T., Polstelnicu, I., Hartmann, M., Stefini, A., Geiser-Elze, A., Gerhold, M., Horn, H., \& Winkelmann, K. (2005). Zur Wirksamkeit psychodynamischer Kurzzeitpsychotherapie bei Kindern und Jugendlichen mit Angststörungen. Praxis für Kinderpsychologie und Kinderpsychiatrie, 54,559-577.

Kurock, V. (2014). Übereinstimmung zwischen Therapeutenund Patientenzielen in der analytischen Kinder- und Jugendpsychotherapie. Unveröffentlichte Bachelorarbeit. MSH Medical School Hamburg.

Laezer, K. L., Tischer, I., Gaertner, B., \& Leuzinger-Bohleber, M. (2015). Aufwendige Langzeit-Psychotherapie und kostengünstige medikamentengestützte Verhaltenstherapie im Vergleich - Ergebnisse einer gesundheitsökonomischen Analyse der Behandlungskosten von Kindern mit der Diagnose ADHS und Störung des Sozialverhaltens. Gesundheitsökonomie und Qualitätsmanagement, 20, 178-185.

Mäß, M. (2014). Ein Vergleich der Therapieziele von Therapeut und Eltern des Patienten bei analytischer Kinder- und Jugendpsychotherapie. Unveröffentlichte Bachelorarbeit. MSH Medical School Hamburg.

Midgley, N., \& Kennedy, E. (2011). Psychodynamic psychotherapy for children and adolescents: a critical review of the evidence base. Journal of Child Psychotherapy, 37, 232-260.

Schepank, H. (1995). Der Beeinträchtigungs-Schwere-Score. BSS; ein Instrument zur Bestimmung der Schwere einer psychogenen Erkrankung; Manual. Göttingen:Beltz Test.

Schulte-Bahrenberg, T. (1990). Therapieziele, Therapieprozess und Therapieerfolg. Pfaffenweiler:Centaurus.

Stefini, A., Geiser-Elze, A., Hartmann, M., Horn, H., Winkelmann, K., \& Kronmüller, K.-T. (2008). Bindungsstil und Therapieerfolg in der psychodynamischen Kurzzeittherapie bei Kindern und Jugendlichen. Psychotherapie und psychologische Medizin, 59, 68-74.
Steinhausen, H.-C. (2006). Psychische Störungen bei Kindern und Jugendlichen. Lehrbuch der Kinder- und Jugendpsychiatrie und -psychotherapie (6. Aufl.). München: Urban \& Fischer.

Trowell, J., Joffe, I., Campbell, J., Clemente, C., Almqvist, F., Soininen, M., Koskenranta-Aalto, U., Weintraub, S., Kolaitis, G., Tomarar, V., Anastasopoulos, D., Grayson, K., Barnes, J., \& Tsiantis, J. (2007). Childhood depression: a place for psychotherapy. An outcome study comparing individual psychodynamic psychotherapy and family therapy. European Child and Adolescent Psychiatry, 16, 157-167.

Trowell, J., Rhode, M., Miles, G., \& Sherwood, I. (2003). Childhood depression: work in progress. Journal of Child Psychotherapy, 29, 147-170.

Weitkamp, K., Daniels, J. K., Romer, G., \& Wiegand-Grefe, S. (eingereicht). Psychoanalytic psychotherapy for children and adolescents with severe externalizing psychopathology: An effectiveness trial.

Wiegand-Grefe, S., Weitkamp, K., Timmermann, H., \& Romer, G. (2011). Hamburger Studie zur Wirksamkeit psychoanalytischer Behandlungen von Kindern und Jugendlichen. In A. Springer, B. Janta, \& K. Münch (Eds.), Angst (S. 147-160). Gießen: Psychosozial-Verlag.

Winkelmann, K., Hartmann, M., Neumann, K., Hennch, C., Reck, C., Victor, D., Horn, H., Uebel, T., \& Kronmüller, K.-T. (2000). Stabilität des Therapieerfolgs nach analytischer Kinder-und JugendlichenPsychotherapie eine Fünf-Jahres-Katamnese. Praxis der Kinderpsychologie und Kinderpsychiatrie, 49,315-328.

Winkelmann, K., Stefini, A., Hartmann, M., Geiser-Elze, A., Kronmüller, A., Schenkenbach, C., Horn, H., \& Kronmüller, K.-T. (2005). Efficacy of psychodynamic shortterm psychotherapy for children and adolescents with behavioral disorders. Praxis der Kinderpsychologie und Kinderpsychiatrie, 54, 598-614.

Winter, S., Wiegard, A., Welke, M., \& Lehmkuhl, U. (2005). Evaluation mit der Psychotherapie Basisdokumentation für Kinder und Jugendliche: Psy-BaDo-KJ. Ein Instrument zur Qualitätssicherung und Therapieevaluation im Bereich Kinder- und Jugendpsychotherapie. Zeitschrift für Kinder- und Jugendpsychiatrie und Psychotherapie, 33, 113-122. 